\title{
Stereotactic ablative radiotherapy for oligometastatic non-small cell lung cancer
}

\author{
Vivek Verma ${ }^{1}$, Joe Y. Chang ${ }^{2}$ \\ ${ }^{1}$ Department of Radiation Oncology, University of Nebraska Medical Center, Omaha, NE, USA; ${ }^{2}$ Department of Radiation Oncology, University of \\ Texas M.D. Anderson Cancer Center, Houston, TX, USA \\ Correspondence to: Joe Y. Chang, MD, PhD. Department of Radiation Oncology, Unit 97, University of Texas M.D. Anderson Cancer Center, 1515 \\ Holcombe Blvd, Houston, TX 77030, USA. Email: jychang@mdanderson.org. \\ Provenance: This is an invited Editorial commissioned by the Section Editor Lei Deng (PGY-1 Resident of Internal Medicine Jacobi Medical Center, \\ Albert Einstein College of Medicine, USA). \\ Comment on: Iyengar P, Wardak Z, Gerber DE, et al. Consolidative Radiotherapy for Limited Metastatic Non-Small-Cell Lung Cancer: A Phase 2 \\ Randomized Clinical Trial. JAMA Oncol 2018;4:e173501.
}

Submitted Nov 08, 2017. Accepted for publication Nov 12, 2017.

doi: $10.21037 /$ jtd.2017.11.141

View this article at: http://dx.doi.org/10.21037/jtd.2017.11.141

Comprising a large proportion of patients with non-small cell lung cancer (NSCLC), metastatic disease is a major oncologic concern. Consequently, enormous ongoing efforts in both systemic and radiation therapy are specifically aimed at delaying progression and potentially increasing survival over historical treatment with palliative chemotherapy. One particular approach is the concept of stereotactic ablative radiotherapy (SABR) for oligometastatic disease, which refers to administering non-invasive local therapy to limited sites of gross disease in efforts to eradicate the most likely areas of progression and potential sources that generate de novo metastases.

The two best known prospective studies of SABR for oligometastatic NSCLC have been led by MD Anderson Cancer Center (1) and the University of Texas Southwestern (UTSW) (2). The former was a randomized phase II study of SABR (with or without maintenance therapy) versus maintenance therapy or observation alone, in 74 patients with $\leq 3$ metastases (not including the primary) who did not progress following first-line systemic therapy. The SABR arm was associated with a near-tripling in progressionfree survival (PFS) (11.9 vs. 3.9 months, $\mathrm{P}=0.005$ ); this was tolerated well with few higher-grade toxicities. The latter was a single-arm phase II trial of 24 patients with $\leq 6$ metastatic sites (extracranial, including primary disease) treated with SABR and erlotinib, with PFS of
14.7 months and overall survival (OS) of 20.4 months, and demonstration of excellent treatment tolerance.

In the article that accompanies this commentary, a multiinstitutional phase II trial led by investigators at UTSW, 29 patients with $\leq 6$ metastatic sites (extracranial, including primary disease) that did not progress following firstline systemic therapy were randomized to maintenance chemotherapy with or without SABR (3). There were several noteworthy findings. Of the 15 patients in the maintenance-alone arm, 10 (67\%) progressed, 7 (70\%) of which were at the original sites of disease; corresponding figures in the SABR arm ( $\mathrm{n}=14)$ were $4(29 \%)$ progressors and $0(0 \%)$ in-field. Similar to the MD Anderson study, this translated to a near-tripling of PFS (9.7 vs. 3.5 months, $\mathrm{P}=0.01$ ) and premature closure of the trial. With a median follow-up in all patients of 9.6 months, the median OS in the SABR arm was not reached.

Given these encouraging outcomes that are numerically comparable with prior data, it is clear that phase III evaluation is necessary. There are three major randomized trials that have recently opened in the United States (4) and Europe $(5,6)$, all of which measure OS as the primary endpoint. The NRG LU002 study aims to enroll 300 patients with newly-diagnosed metastatic or previously treated NSCLC that develops metastases. Other inclusion criteria are $\leq 3$ sites of extracranial metastases, a performance 
status of $0-2$, and ability to receive re-irradiation in cases of previous treatment. Of note, brain metastases are allowed if previously treated and without progression; untreated and/or progressive ones are excluded. It is also notable that receipt of targeted therapies in the first-line setting is an exclusion criterion. The European SARON study (estimated accrual 340 patients) includes those with $\leq 3$ metastatic areas, negative EGFR/ALK mutations, and performance status $0-1$. Notable exclusions are patients with any prior treatment for NSCLC, and isolated brain metastases without disease elsewhere. The COMET trial has completed accrual (99 patients) and evaluates palliative RT or SABR for up to 5 metastatic lesions ( 3 in any one organ system) in patients with performance status $0-1$, excluding those with femoral bone metastases, intracerebral-only metastases, and prior RT to the same site.

These criteria may be analyzed further in order to comment on optimal patient selection for utilizing SABR for oligometastatic NSCLC in future work. Although all trials correctly include only patients with good performance status (albeit the differing definitions between studies), none use other prognostic factors such as volume of disease and histology $(7,8)$, which would likely hinder accrual. Next, the differing inclusion of patients with intracerebral disease is also interesting, especially in light of their inclusion in the recent UTSW trial. However, recently published data demonstrate that patients with single brain metastasis and limited extracranial metastatic sites (along with favorable age and performance status) can survive for numerically prolonged periods (9). Future trials should also seek to stratify patients with any level of response to chemotherapy, from those with just stable disease. This could be utilized as a surrogate for favorable tumor biology, because oligometastatic disease with favorable biology may be better controlled in-field, with lower risk to progress elsewhere. Although utilizing genomic signatures for these purposes is still under development, it underscores the major challenges in including the most favorable subsets of stage IV NSCLC patients onto the "first wave" of randomized trials. Not only would any benefit to aggressive local therapy presumably lie with those patients, payers will likely scrutinize these phase III studies carefully in order to evaluate the cost-effectiveness of SABR in these circumstances. Although it can be argued both ways whether PFS is a "clinically meaningful" endpoint in the absence of direct OS comparisons, it is crucially important to design trials prudently so that payment ramifications are not a deterrent to future enrollment.

A shortcoming of all studies is the absence of targeted therapies, specifically immunotherapy. The latter is now the first-line treatment in metastatic or recurrent NSCLC based on randomized evidence $(10,11)$. Although this necessitates design by future trials to account for this parameter, it is currently uncertain whether the benefits of aggressive local therapy would be "diluted" by more beneficial effects of immunotherapy over cytotoxic chemotherapy. However, there are reasons to believe that the impact of SABR could be even greater in the presence of immunotherapy (12). This is largely owing to SABR-immunotherapies promoting abscopal responses against known areas of disease but potentially also areas of subclinical or micrometastases $(13,14)$. To this extent, an ongoing randomized phase II trial of stage I or isolated recurrence of NSCLC is evaluating SABR alone versus SABR-immunotherapy (nivolumab) (termed I-SABR) (15). In light of this information, it is important to consider that all of the accruing phase III trials (unlike currently published data) allow for hypofractionated or conventionally-fractionated radiotherapy. Not only may these have inferior local control compared to ablative dosing (which is a prespecified secondary endpoint in all studies), non-ablative regimens cannot induce abscopal responses as much as SABR. Although SABR will likely be the dominant radiotherapy modality in those trials, inclusion of a high proportion of non-ablative radiotherapy delivery may diminish effects on multiple endpoints. Although this statement remains purely speculative, stratification should ideally be performed for this parameter.

Similar to immunotherapy, targeted therapies are also under-studied in prior work on this topic. The recently published trial in comment excluded patients with EGFR/ALK mutations, and although the prior single-arm phase II from UTSW specifically administered erlotinib to all patients (2), measurement of EGFR status was neither a requirement nor performed in the majority of patients. This is also important in light of the aforementioned data on prognostic markers for NSCLC brain metastases, which have now included EGFR and ALK mutational status (8). Although targeted therapies for these mutations are often used in the first-line for appropriate patients, performing up-front SABR in well-selected oligometastatic patients may produce outcomes at least comparable to the encouraging results of multiple oligometastases trials discussed above. In other words, these patients may prove 
to be a good-prognostic subset for which aggressive ablative local therapy may provide proportionally higher benefits. Although high-level data to support this notion are lacking, this must be a factor addressed in future multi-institutional and prospective work. An ongoing randomized trial (primary endpoint PFS) is evaluating EGFR mutant disease, not progressing following osimertinib, to continued osimertinib versus local therapy followed by osimertinib (16).

Taken together, the work of Iyengar and colleagues offers an improved glimpse of the utility of SABR for oligometastatic NSCLC; it validates that in well-selected patients, local therapy decreases risk of progression in areas that are initially present (3). In context of currently accruing phase III trials, the utility of immunotherapy with SABR is highly novel and represents an area that, with supportive data, could prove to be truly practice-changing. However, patient selection is crucial, and the optimal applicability of these regimens will likely remain elusive and without consensus for the foreseeable future.

\section{Acknowledgements}

None.

\section{Footnote}

Conflicts of Interest: The authors have no conflicts of interest to declare.

\section{References}

1. Gomez DR, Blumenschein GR Jr, Lee JJ, et al. Local consolidative therapy versus maintenance therapy or observation for patients with oligometastatic non-smallcell lung cancer without progression after first-line systemic therapy: a multicentre, randomised, controlled, phase 2 study. Lancet Oncol 2016;17:1672-82.

2. Iyengar P, Kavanagh BD, Wardak Z, et al. Phase II trial of stereotactic body radiation therapy combined with erlotinib for patients with limited but progressive metastatic non-small-cell lung cancer. J Clin Oncol 2014;32:3824-30.

3. Iyengar P, Wardak Z, Gerber DE, et al. Consolidative radiotherapy for limited metastatic non-small-cell lung cancer: a phase 2 randomized clinical trial. JAMA Oncol 2017;4:e173501.

4. Maintenance chemotherapy with or without stereotactic body radiation therapy in treating patients with stage
IV non-small cell lung cancer. Available online: https:// clinicaltrials.gov/show/NCT03137771. Accessed October 29, 2017.

5. Stereotactic ablative radiotherapy for oligometastatic nonsmall cell lung cancer (SARON). Available online: https:// clinicaltrials.gov/show/NCT02417662. Accessed October 29, 2017.

6. Stereotactic ablative radiotherapy for comprehensive treatment of oligometastatic tumors (SABR-COMET). Available online: https://clinicaltrials.gov/ct2/show/ NCT01446744. Accessed November 6, 2017.

7. Milano MT, Katz AW, Zhang H, et al. Oligometastases treated with stereotactic body radiotherapy: long-term follow-up of prospective study. Int J Radiat Oncol Biol Phys 2012;83:878-86.

8. de Vin T, Engels B, Gevaert T, et al. Stereotactic radiotherapy for oligometastatic cancer: a prognostic model for survival. Ann Oncol 2014;25:467-71.

9. Sperduto PW, Yang TJ, Beal K, et al. Estimating survival in patients with lung cancer and brain metastases: an update of the graded prognostic assessment for lung cancer using molecular markers (Lung mol-GPA). JAMA Oncol 2017;3:827-31.

10. Herbst RS, Baas P, Kim DW, et al. Pembrolizumab versus docetaxel for previously treated, PD-L1-positive, advanced non-small-cell lung cancer (KEYNOTE-010): a randomised controlled trial. Lancet 2016;387:1540-50.

11. Reck M, Rodriguez-Abreu D, Robinson AG, et al. Pembrolizumab versus chemotherapy for PD-L1positive non-small-cell lung cancer. N Engl J Med 2016;375:1823-33.

12. Bernstein MB, Krishnan S, Hodge JW, et al. Immunotherapy and stereotactic ablative radiotherapy (ISABR): a curative approach? Nat Rev Clin Oncol 2016;13:516-24.

13. Reynders K, Illidge T, Siva S, et al. The abscopal effect of local radiotherapy: using immunotherapy to make a rare event clinically relevant. Cancer Treat Rev 2015;41:503-10.

14. Verma V, Lin SH. Implications of the bystander and abscopal effects of radiation therapy. Clin Cancer Res 2016;22:4763-5.

15. Clinical Trials Comparing Immunotherapy Plus Stereotactic Ablative Radiotherapy (I-SABR) Versus SABR Alone for Stage I, Selected Stage IIa or Isolated Lung Parenchymal Recurrent Non-Small Cell Lung Cancer: I-SABR. Available online: https://clinicaltrials.gov/ct2/ show/NCT03110978. Accessed November 6, 2017. 
16. National Comprehensive Cancer Network. NCCN News What's Next? NCCN Gathers Healthcare Policy Experts to Deliberate Challenges to Patient Safety and Access to Cancer Care Under the New Administration NCCN and Cancer LinQ Collaborating to Provide Evidence-

Cite this article as: Verma V, Chang JY. Stereotactic ablative radiotherapy for oligometastatic non-small cell lung cancer. J Thorac Dis 2018;10(1):21-24. doi: 10.21037/jtd.2017.11.141
Based, Decision-Making Resources to Physicians NCCN Awards Grants to Investigators at Member Institutions to Study Osimertinib in Lung Cancer. Available online: http://www.jnccn.org/content/15/7/xxviii.extract. Accessed November 6, 2017. 